\title{
A STUDY OF THE INFLUENCE OF THE CHARACTER OF AN EXAMINING ROOM ON THE PERIPHERAL BLOOD VESSELS OF NORMAL, HYPERTENSIVE, AND SENILE SUBJECTS ${ }^{1}$
}

\author{
By C. NEUMANN, A. E. COHN, ANd G. E. BURCH ${ }^{2}$ \\ (From the Hospital of the Rockefeller Institute for Medical Research, New York)
}

(Received for publication February 6, 1942)

The decor, the very furnishings of a room, have turned out to be decisive in the results of examinations of certain kinds of physiological behavior. Circulatory reactions which did not occur or occurred to a less or slight degree in a room equipped like a laboratory, were more extensive and more nearly uniform in a room furnished like an ordinary bedroom. The results of investigations intended to describe "basal" conditions, whether psychological or physiological in a more general sense, may all be subject to changes away from familiar environments. The fact that the environment in which such studies are undertaken influences the result is well known and has no doubt received attention both from physiologists, as from Pavlov in the case of animals, and from psychologists, both practical in the case of decorators, and academic in the schools. Although known, there is scarcely a reference to the bearing of this factor upon the results of such investigations as are now being reported.

These studies were concerned with quantitative measurements of spontaneous variations in the volumes of fingers and toes and of the time of reaction (constriction) of small blood vessels following the application of sensory stimuli at distant parts of the body $(1,2)$.

The apparatus consisted of a camera by means of which the movements of a sensitive membrane, activated by the pulsations of a finger tip, could be recorded. The only part of the apparatus in contact with the patient was a lightweight chamber which fitted loosely over the terminal portion of the tip of a fingep or toe. Application of a resinous material was relied upon to seal the chamber in place. The tips of fingers and toes of all patients underwent rhythmic variations in volume, which were recorded as a series

1 This is the 7th paper reporting the results of studies of the small blood vessels and related subjects.

2 Commonwealth Fund Fellow. of waves whose upward deflections signified increase in volume of the part, and downward deflections, a decrease. For the most part, such variations in volume occurred without any obvious cause. It was soon learned, however, that downward deflections could be occasioned by subjecting the patient to a sensory or ideational stimulus. The present report is an attempt to ascertain some of the conditions which allow such decreases in volume to take place, and is presented separately from a more detailed description of the purely mechanical aspects of obtaining the data in view of the unexpected new information which was uncovered, not by improvement in technique, but rather by closer control of the environmental aspects of the experiment.

Our experiments were first carried out in a medium-sized air-conditioned laboratory, furnished with a typical hospital bed, two or three small chairs, and a mass of unfamiliar equipment such as a large laboratory sink and a table on which were kept unused pieces of apparatus. The recording capsule, camera, and timers were screened from the patient by forbidding black, reasonably light-tight curtains, through which he could hear distinctly noises and clicks incident to the use of the apparatus. He was always aware of the presence of the observers, even though he could not see them. The apparatus for the application of stimuli (heat, cold, pin-prick, touch) was hidden also by a curtain. The observer was separated from the subject by still another curtain. At no time could he predict the moment of application of a stimulus. Person after person commented upon the fact that he felt himself under a state of considerable "tension" and apprehension. The darkened "laboratory", the type of room, the knowledge that persons were working immediately behind screens near his bed, and the anticipation of something unexpected contributed to the state of his feeling. Some patients disarmed 
even under these circumstances but that was the exception.

Obviously, the most desirable time to carry out observations like these is when individuals regard themselves as being, and are, in a state of relaxation. Care has not usually been taken in such studies to see to it that these conditions are actually attained. Accordingly, certain changes were made in our examining room in the hope of putting subjects at greater ease. The laboratory was divided. One part remained laboratory; the other part became bedroom. A one-way mirror permitted the observation of patients from the laboratory. The bed and walls were draped with material of a pleasing design. The room was furnished with a small bed-table, a Morris chair, rugs, lamps, magazines, coat rack, and pictures. A subject entered, not a frightening laboratory equipped with strange machines, but was conducted into a comfortable room, not too dissimilar from his own. He came into contact not with a staff of observers but with one physician only who put plethysmographic cups in place and explained that they led to the rest of the apparatus located in a distant part of the building. The records were in fact made in the adjoining laboratory by an observer who could see and hear what was going on. Communication between the observers though separated only by a wall was carried on by telephone to create the illusion of distance. The stimuli were not applied until the patient had been left alone and was resting quietly. As stimulus, use was made of a telephone-like bell rather than of an unfamiliar noise, and for light, a bright diffuse light. Both stimuli were set in operation from the adjoining room. When it came time to stimulate with heat, cold, or pin-pricks, the first observer reentered the bedroom and raised a small white curtain between the subject and himself. It would have been better to apply all stimuli mechanically but this method was adopted to avoid the need of affixing appliances to the subject's body.

In the original "laboratory", it was noticed that most of the normal subjects "relaxed" and rested comfortably while the patients with hypertension did not. It was found also in 15 normal subjects that 160 stimulations were followed by 117 (73 per cent) instances of vasoconstriction, while in 13 patients with essential hypertension, vasoconstriction was less frequent (102 of 224 stimuli or 42 per cent). In the 10 senile subjects, this reaction occurred in 46 of 107 stimulations (43 per cent) (Table I). The normal subjects were either technicians or were otherwise familiar with laboratories. This was not the case with the patients with hypertension and the senile subjects. It was noticed that if recordings were obtained without the subject's knowledge, he became more "relaxed" as evidenced by the increase in size of the pulse and alpha waves $(1,2)$, and developed vasoconstriction more readily following stimulation. In any event, everyone subjected to the tests frequently commented unfavorably upon the apparatus, curtains, darkness of the laboratory, small beams of light, and the presence and movements of the observers. Of them all, hypertensive patients were most offended.

TABLE I

The effects of environmental conditions upon the incidence of vasoconstriction in the blood vessels of the finger tips of normal and senile subjects and patients with hypertension following the application of sensory stimuli to another part of the body

\begin{tabular}{|c|c|c|c|c|c|c|}
\hline \multirow{2}{*}{ Subjects } & \multirow{2}{*}{ Number } & \multirow{2}{*}{ Type of room } & \multicolumn{2}{|c|}{$\begin{array}{l}\text { Number of responses of blood vessels } \\
\text { to application of a sensory stimulus }\end{array}$} & \multirow{2}{*}{ Total stimuli } & \multirow{2}{*}{$\begin{array}{l}\text { Per cent of stimul } \\
\text { followied by } \\
\text { vasoconstriction }\end{array}$} \\
\hline & & & Vasoconstriction & No response & & \\
\hline \multirow{2}{*}{ Normal } & \multirow{2}{*}{15} & Laboratory & 117 & 43 & 160 & 73 \\
\hline & & Bedroom & 44 & 19 & 63 & 70 \\
\hline \multirow{2}{*}{ Hypertensive } & \multirow{2}{*}{13} & Laboratory & 102 & 142 & 244 & 42 \\
\hline & & Bedroom & 22 & 12 & 34 & 65 \\
\hline \multirow{2}{*}{ Senile } & \multirow{2}{*}{10} & Laboratory & 46 & 61 & 107 & 43 \\
\hline & & Bedroom & 32 & 20 & 52 & 62 \\
\hline
\end{tabular}




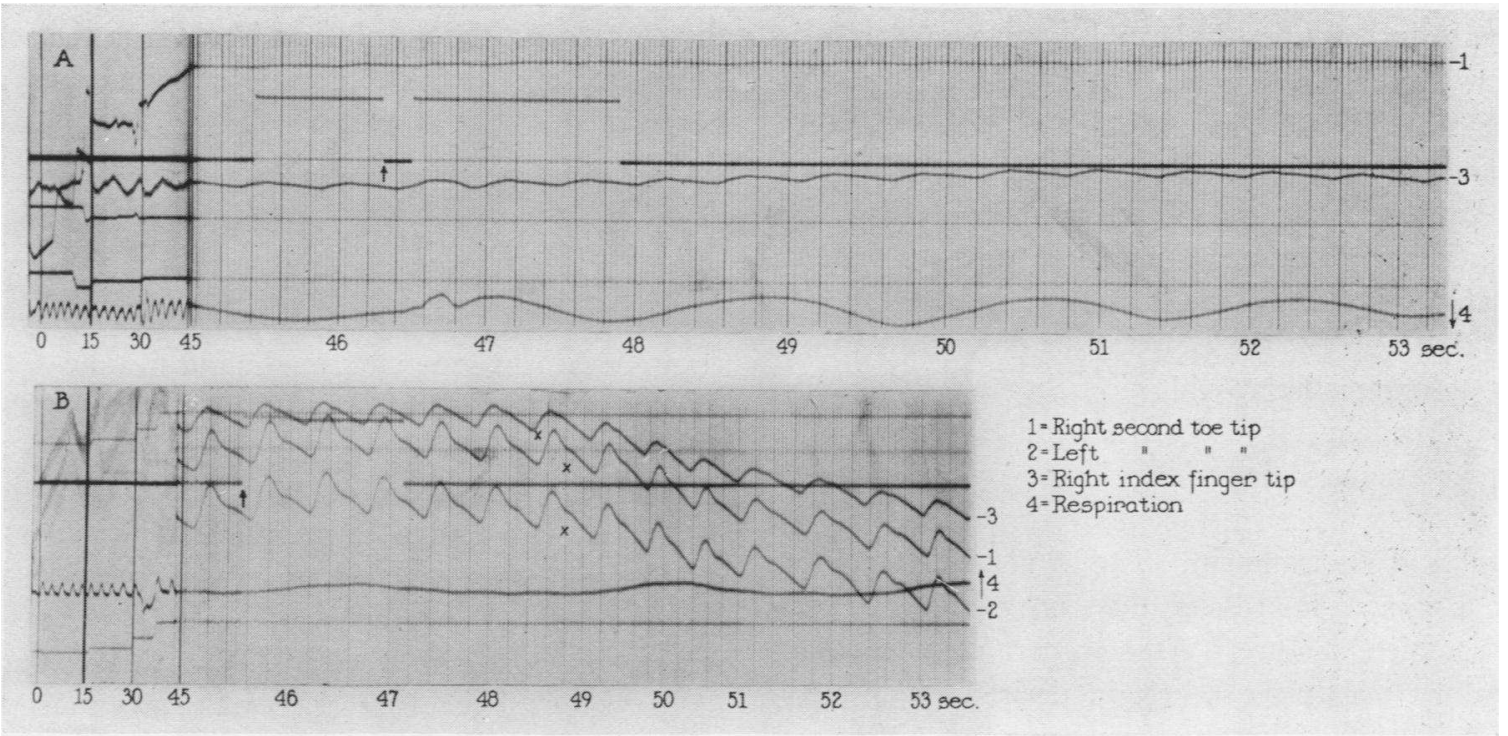

Fig. 1. Plethysmographic Records of the Tips of the Fingers and Toes of a Patient WiTh HYPERTENSION

Senory stimuli were applied at the arrows. The pulse and alpha waves are on the same scale.

A. A representative record is shown taken during one of 11 separate observations on the subject in the "laboratory" type of environment. The blood vessels of the finger and toe tips are spastic as indicated by the small size of the pulse and alpha waves before the application of the stimulus.

B. A representative record is exhibited taken during the first observation in the bedroom. The vessels are much less constricted than in Figure 1A. The vasoconstriction began at the points marked by $\mathrm{X}$, about 3.3 seconds after the application of the stimulus.

After the laboratory was changed and the new room put into use, the frequency of vasoconstriction following stimulation increased markedly in the patients with hypertension and the senile subjects, equalling the incidence in normal persons. In the senile subjects, vasoconstriction occurred in 32 of 52 (62 per cent) trials; in the patients with hypertension, in 22 of 54 (65 per cent); and in 44 of 63 (70 per cent) in normal individuals, that is to say, 73,42 , and 43 per cent as against 70, 65 and 62 . Everybody avowed voluntarily that he found the new environment to be comfortable, conducive to relaxation and that he was much less apprehensive shortly after having been left alone.

The behavior of 2 patients with hypertension deserves separate comment. In the "laboratory," they remained "tense" and very apprehensive on 10 and 11 separate observations, respectively, spread over a period of 3 months. It was with great difficulty that a few reactions were obtained. But on the first trials in the new environmental conditions, they "relaxed"; practically every stimulus was followed by vasoconstriction (Figure 1 ).

\section{COMMENT}

The fact that relaxation occurred derives its confirmation from two circumstances. First, from the statements of subjects, practically all of whom asserted they felt more comfortable and under less tension in the bedroom than in the laboratory where they could not but feel acutely that they were being experimented upon. And second, from the appearance of the subjects and from the plethysmographic records themselves. In general, when the pulse waves were small, a stimulus would not bring about much, if any, vasoconstriction; but when $\operatorname{largc}$, except in a few senile subjects, the likelihood of vasoconstriction was greatly increased. It was very common for hypertensive subjects, in particular, to exhibit small pulse waves in the "laboratory" and large ones when the room was made pleasant. At times, patients would volunteer the information that they felt relaxed in the "laboratory". Without exception, examination would then reveal that the pulse waves were large.

Vasoconstriction consists of two factors, de- 
crease in the size of the pulse waves and also in that of the alpha waves. It is a special type of the latter which documents the major decrease when the subject reacts to a sensory stimulus (2).

Even without the subjective and objective data which have been accumulated to support the idea that a familiar room is more agreeable than a strange one for these particular purposes, subject or observer alike would select the new rather than the old room, especially if the observer wished to avoid as many disturbing factors as possible.

There is another factor which may decide the choice of environment. The avoidance of pain or of a painful impression does not necessarily give a complete picture. A room arranged as a laboratory may place a certain desired strain upon individuals and by so doing, bring out differences which might otherwise have been overlooked.

Although the hypertensive and senile subjects showed a higher percentage of reactions in the bedroom than in the laboratory, no such difference was noticed in the normal subjects. But our "normal" subjects were, as noted above, acquainted with the hospital and were familiar even with the particular laboratory used. The changes in the room could hardly have made much difference to them. However, the frequency of reactions was raised in the hypertensive and senile subjects approximately to the level of the normal group by just a few simple changes in the environment. Had the old room continued to be in use, the conclusion would have been drawn that there was a difference in the three groups of subjects, but in the new room, the conclusion is obvious that the same reaction is possible and took place in all groups.

\section{SUM M ARY}

Objective evidence supports the belief that the conditions under which physiological studies are carried out must be suitably arranged, not only to assure uniform temperature, humidity, and state of digestion, but also less tangible factors such as the patient's mental comfort and the degree of his relaxation. This was demonstrated by converting a "laboratory" into a conventional bedroom and by observing how the frequency of reaction on the part of peripheral blood vessels increased when sensory stimuli were applied at distant parts of the body. This observation was made not only in the case of groups of hypertensive and senile subjects but also in individual subjects studied under both types of environment. Conversely, in tense individuals, to be unable to relax in the atmosphere of a "laboratory" is evidence of the possible presence of an abnormal process.

\section{REFERENCES}

1. Burch, G. E., Cohn, A. E., and Neumann, C., A study by quantitative methods of the spontaneous variations in volume of the finger tip, toe tip, and postero-superior portion of the pinna of resting normal white adults. Am. J. Physiol., 1942, 136, 433.

2. Burch, G. E., Cohn, A. E., and Neumann, C., Reactivity of intact blood vessels of the fingers and toes to sensory stimuli in normal resting adults, in patients with hypertension, and in senile subjects. J. Clin. Invest., 1942, 21, 655. 